\title{
Prácticas parentales y entorno de residencia en adolescentes: un estudio comparativo a nivel de riesgo psicosocial
}

\section{Parenting practices and neighborhoods in adolescents: a comparative study at the level of psychosocial risk}

\author{
Alvaro Jaime Hinostroza Ballón ${ }^{1 a}$ \\ Universidad Peruana de Ciencias Aplicadas. Lima, Perú $^{1}$ \\ (iD) Orcid ID: https://orcid.org/0000-0002-4853-6707 ${ }^{1}$
}

Recibido: 02 de setiembre de 2020

Aceptado: 24 de noviembre de 2020

\begin{abstract}
Resumen
El presente estudio se realiza con la finalidad de describir y comparar los resultados entre 85 adolescentes que residen en una zona considerada de alto riesgo psicosocial del Callao (Perú) con un grupo de 82 adolescentes de similares características sociodemográficas que reside en una zona no considerada de alto riesgo psicosocial de la misma jurisdicción. La metodología es de tipo descriptivo - comparativo y de diseño no experimental, transversal. En este caso, se usó la Escala de Afecto y la Escala de Normas y Exigencias, y la Escala de Violencia en el Barrio. Los resultados permiten afirmar que los adolescentes de una zona de alto riesgo psicosocial expresan, desde su percepción, que sus padres priorizan el afecto, el interés y la comunicación, atribuyéndole especialmente al objeto materno dicha práctica. De la misma manera, refieren también, que sus padres recurren al diálogo cuando deben de supervisar o exigir el cumplimiento de sus límites; sin embargo, a comparación del grupo control, evalúan a sus padres de manera más autoritaria.
\end{abstract}

Palabras clave: Prácticas parentales, percepción, adolescencia, riesgo psicosocial.

\begin{abstract}
The present study is carried out with the purpose of describing and comparing the results between 85 adolescents who reside in an area considered to be of high psychosocial risk in the city of Callao (Peru) with a group of 82 adolescents with similar sociodemographic characteristics who reside in an area not considered to be of high psychosocial risk of the same jurisdiction. The methodology is descriptive - comparative and has a non-experimental, crosssectional design. In this case, the Escala de Afecto and the Escala de Normas y Exigencias and the Escala de Violencia en el Barrio were used. The results allow us to establish that adolescents in a zone of high psychosocial risk express, from their perception, that their parents prioritize affection, interest and communication, and they attribute this practice especially to the maternal
\end{abstract}

${ }^{\mathrm{a} C}$ Correspondencia al autor

E-mail: alvaro.hinostroza@gmail.com 
object. Similarly, they also refer, that their parents resort to dialog when they must monitor or demand compliance with their limits; however, compared to the control group, they evaluate their parents more authoritatively.

Keywords: Parenting practices, perceived, adolescence, psycho-social risk

\section{Introducción}

La adolescencia comprende la etapa etaria que presenta mayor cantidad de cambios (emocionales, físicos, fisiológicos, etc.). En este caso, existen diversas propuestas teóricas que describen y justifican las conductas presentes en esta etapa; sin embargo, la principal fuente de información debe surgir del propio adolescente. Es pues, labor de quien pretenda ejercer influencia en ellos, estar atento a lo que nos comunican y a los permanentes riesgos que están expuestos. La intensidad con que perciben las emociones y la necesidad del adolescente de individualizarse de sus padres, lo condiciona a explorar vivencias nuevas y asumir peligros (Benarous, et al., 2020; Benarous \& Mazet, 2020; Bernal \& Bernal, 2020; Bonilla, 2020; García-Álvarez et al., 2020). Por lo tanto, diseñar e implementar políticas preventivas en favor de los jóvenes es una acción necesaria.

Se reconoce al constructo prácticas parentales como la vía que teoriza las conductas específicas de los padres para poder dimensionarse y categorizarse bajo algún modelo. De acuerdo a Darling y Steinberg (1993), el estilo de crianza es la suma de las actitudes de los padres que se transmiten y expresan a los hijos a través de comportamientos paternos específicos, a lo que se denomina prácticas parentales, y/o a través de comportamientos paternos no específicos como gestos, modulaciones de la voz o expresiones espontáneas de las emociones percibidas. Lo cual es ratificado por diferentes estudios (Catemaxca, 2018; Cruz, Flores \& Jiménez, 2019; Espinosa, Mendoza \& Villalba, 2020), quienes refieren que las prácticas parentales son las acciones concretas que los adultos encargados realizan para asegurar e impulsar el desarrollo del menor de manera que logre aprender a desenvolverse autónoma y socialmente en el mundo que lo rodea. Asimismo, Catemaxca, 2018; Cruz, Flores y Jiménez, 2019; Espinosa, Mendoza y Villalba 2020, han demostrado la asociación entre las prácticas parentales, medido desde el apoyo y control parental, con el desarrollo integral del niño y adolescente debido a que es determinante para la salud integral de cada infante.

Según Díaz y Moral (2018); Ibarra (2020); Mendoza, Claros y Peñaranda (2016) la adolescencia intermedia, que se estima entre los 14 y 16 años, es la etapa en la que los conflictos sociales prevalecen, así como su interés en distanciarse de las normas y acompañamiento 
paterno. El proceso de aceptación del cuerpo debe concluir y buscan mostrarse atractivos, lo cual corresponde con disposición a vivenciar experiencias de intimidad sexual. Se intensifican sus emociones e impulsos, experimentan una sensación de omnipotencia y el grupo de pares asume la estructura de normas y códigos de conducta, por ello es la etapa en que están más expuestos a conductas de riesgo. En tal sentido, es un período de alta vulnerabilidad psicológica, en el que es frecuente la aparición de comportamientos de riesgo o trastornos mentales (Álvarez-Malé, Bautista \& Serra, 2015; Díaz \& Moral, 2018; Ibarra, 2020; Martín-Fernández, Matalí, García-Sánchez, Pardo, Lleras, Castellano-Tejedor, 2017).

Las prácticas parentales dirigidas a manifestar afecto y a conseguir control sirven como factores protectores y reguladores de conducta ante los conflictos que el adolescente pueda vivenciar. Ello se corrobora en las diversas investigaciones que reconocen la trascendencia de las prácticas parentales en el ajuste conductual durante la etapa adolescente (Catemaxca, 2018; Cruz, Flores \& Jiménez, 2019; Espinosa, Mendoza \& Villalba, 2020).

Diversas investigaciones (Chetty, Hendren \& Katz, 2016; Madueño, Lévano \& Salazar, 2020; Newbury, Arseneault, Caspi, Moffitt, Odgers \& Fisher, 2016) han señalado la asociación significativa y positiva entre un entorno de residencia de alto riesgo, con problemas de conducta en la niñez y consecuente incursión en conductas de riesgo durante la adolescencia. Considerando, además, la trascendencia que tiene para el ajuste conductual del adolescente las prácticas parentales empleadas en su crianza, los investigadores (Del Barrio et al., 2009; Cuellar, Jones \& Sterrett, 2015; Suen, et al., 2019) han evaluado y aseverado el poder mediador de las prácticas parentales para canalizar la influencia de los entornos de residencia.

De esta manera, considerando la trascendencia de la crianza en la socialización de la persona y la necesidad de la sociedad de proteger a sus menores en riesgo; es que la presente investigación pretende describir las prácticas parentales percibidas de los padres por un grupo de adolescentes que viven en una zona de alto riesgo psicosocial y compararlas con otro grupo de adolescentes que vive en una zona no considerada de alto riesgo psicosocial; a través del constructo prácticas parentales y las dos dimensiones de crianza que hasta la actualidad generan representatividad en la literatura científica por su trascendencia en el desarrollo formativo de un hijo: el apoyo y el control parental.

Este estudio se centra en la Provincia Constitucional del Callao, en donde el Observatorio de la Criminalidad del Callao (2015) presentó lo que ellos denominaron el "Mapa del delito del Callao", en donde se evidencia qué tipo de delitos se han cometido en cada distrito desde enero a octubre del 2015, permitiendo así identificar los entornos de residencia de mayor riesgo. Específicamente, los resultados acerca de los 2 distritos planteados por la presente investigación 
como zona no considerada de alto riesgo (La Perla) y zona considerada de alto riesgo (CallaoCercado) avalan nuestra elección.

\section{Método}

\section{Diseño}

El presente estudio es de tipo descriptivo - comparativo dado que se pretende analizar las prácticas parentales y el entorno de residencia en dos grupos de adolescentes. De la misma manera, se trata de un diseño no experimental ya que las variables no han sido controladas. Asimismo, es un diseño de investigación de tipo transversal, ya que se recogen los datos de ambos grupos en un solo momento, sin realizar un seguimiento de la muestra (Fresno, 2019).

\section{Participantes}

En la selección de la muestra se utilizó un tipo de muestreo no probabilístico y accidental. Bajo estos parámetros, la muestra estuvo conformada por 167 adolescentes: 85 adolescentes (51 mujeres y 34 varones) entre los 14 y 15 años de edad $(M=14.31 ; D E=0.47)$ que viven y estudian en una zona considerada de alto riesgo psicosocial de la Provincia Constitucional del Callao y 82 adolescentes (42 mujeres y 40 varones), del mismo rango de edad, $(M=14.63 ; D E$ $=0.48)$ que viven y estudian en una zona no considerada de alto riesgo psicosocial de la Provincia Constitucional del Callao.

\section{Instrumentos}

El primer instrumento utilizado en la presente investigación es la Escala de Afecto: Versión para hijos (EA-H), elaborada por Bersabé, Fuentes y Motrico (2001) para evaluar el constructo apoyo parental mediante el estudio de dos factores: el factor I afecto-comunicación, que mide el afecto, interés y comunicación que los padres manifiestan con sus hijos; y el factor II crítica-rechazo, que evalúa la crítica, el rechazo y la falta de confianza que los hijos perciben de sus padres. La EA-H está compuesta por 20 ítems, donde 10 ítems evalúan cada factor. Cada factor obtiene un puntaje entre 10 y 50 mediante un formato Likert. En cuanto a la confiabilidad del instrumento, la evaluación de la figura materna correlaciona con el Factor I en .87 y con el Factor II en .81; mientras que la evaluación de la figura paterna correlaciona con el Factor I en .90 y con el Factor II en .83. De acuerdo a la validez del instrumento, Bersabé et al. (2001) sustentan la validez de la EA-H mediante el tipo de validez convergente. La correlación entre la puntuación total de afecto obtenida con el EA-H y la puntuación total hallada con el Inventory of Parent and Peer Attachment (IPPA) de Armsden y Greenberg que evalúa de manera conjunta 
el nivel de afecto con padres y grupo cercano de pares, resulta bastante elevada: 0.76 en lo referido a la evaluación de las prácticas parentales maternas y 0.77 en lo referido a la evaluación de las prácticas parentales paternas.

El segundo instrumento utilizado es la Escala de Normas y Exigencias: Versión para hijos (ENE-H), fue elaborada también por Bersabé et al. (2001) para evaluar el constructo control parental mediante el estudio de tres factores: el factor 1 forma inductiva, que contempla una actitud dialogante de los padres al definir, justificar y supervisar el cumplimiento de determinados límites; el factor 2 forma rígida, en donde la actitud paterna predominante en el momento de ejercer autoridad es la imposición y lo establecido puede ser considerado como muy riguroso para un niño; y el factor 3 forma indulgente, cuando por la permisividad de los padres no se consiguen establecer límites, o en todo caso, evaden la supervisión de que lo acordado se cumpla.

La ENE-H está compuesta por 28 ítems: 10 ítems corresponden al factor 1, 10 ítems al corresponden al factor 2 forma rígida, y 8 ítems corresponden al factor 3 forma indulgente. En los dos primeros factores se pueden obtener calificaciones no menores a 10 y no mayores a 50 puntos, mientras que la calificación del tercer factor fluctúa entre 8 y 40 puntos. Los ítems se presentan en un formato de respuesta Likert. En cuanto a la confiabilidad del instrumento, la evaluación de la figura materna correlaciona con el Factor 1 en .80, con el Factor 2 en .72 y con el Factor 3 en .64; mientras que la evaluación de la figura paterna correlaciona con el Factor 1 en .85 , con el Factor 2 en .73 y con el Factor 3 en .60. De acuerdo a la validez del instrumento, Bersabé et al. (2001) sustentan la validez de la ENE-H mediante el tipo de validez convergente. Al analizar la correlación con el Parental Authority Questionnaire (PAQ) de Buri (1991), las autoras comprobaron la adecuada correlación entre ambos constructos: $0.50,0.56$ y 0.40 en lo referido a la evaluación de la madre, y $0.63,0.56$ y 0.39 en lo referido a la evaluación del padre.

El tercer instrumento utilizado es la Escala de violencia en el barrio, la cual es la versión adaptada para España por Gracia, Fuentes y García (2010), del postulado teórico Perceived neighborhood violence propuesto por Robert Sampson, Stephen Raudenbush y Felton Earls en 1997. Los autores afirman que la ocurrencia de alguna de las situaciones implicaría 1 punto, por lo que la escala tendría una puntuación global de violencia percibida en el barrio entre 0 y 5 puntos. La escala fue empleada en inglés por Gracia, Herrero, Fuente y Lila (2008, 2009), Sampson y Raudenbush (1999), y en español para población colombiana (Duque y Klevens, 2000) y población española (Fuentes et al., 2015) en sus respectivas investigaciones sobre la violencia en la comunidad. La escala sólo tiene una puntuación global, por lo que el coeficiente Alpha de Cronbach global fue de 0.711 y 0.72 respectivamente. 


\section{Consideraciones éticas}

La aplicación se dio previa coordinación con cada director de la Institución escogida. A cada padre de familia se le entregó un consentimiento informado donde adquirían conocimiento del propósito de la intervención y aceptaban la evaluación en la hora de tutoría. El día de la aplicación se contó con la participación de un profesional en psicología como apoyo y se acudió con los protocolos individuales agrupados en un sobre manila. Se explicó a los estudiantes la manera en que debían responder la ficha y los cuestionarios; solicitándoles que inicien completando la Ficha sociodemográfica, continúen con el documento titulado Escala de Afecto, culminen con la Escala de Normas y Exigencias. Al finalizar, cada estudiante entregó el sobre con los protocolos resueltos en su interior. No excedieron los 30 minutos esperados y no se presentaron interrupciones que puedan interferir en los resultados, por lo que la aplicación se dio sin contratiempos.

\section{Análisis de datos}

Los resultados obtenidos fueron analizados mediante el paquete estadístico SPSS. En primer lugar, se analizó la normalidad de las puntuaciones mediante la prueba KolmogorovSmirnov dado el número de muestra de 167 participantes. Los puntajes mostraron distribución no normal, por lo que en todos los análisis se utilizaron estadísticos no paramétricos.

Luego, se realizaron análisis descriptivos en cuanto a la tendencia central y de dispersión del grupo para observar la variabilidad de las puntuaciones en el grupo estudiado y se procedió a efectuar el análisis comparativo de medias con 2 variables independientes usando U de Mann Whitney. El análisis de correlación se dio usando el coeficiente Spearman; para finalmente determinar, la probabilidad de no cometer un error tipo II y la magnitud del efecto mediante el hallazgo del valor de la potencia y de la d de Cohen, respectivamente.

\section{Resultados}

A continuación, se procede a presentar los resultados obtenidos considerando los objetivos de la investigación. En primer lugar, atendiendo el objetivo general, se muestra el análisis de diferencias de las prácticas parentales de acuerdo a la figura parental y el análisis de diferencias de las prácticas parentales de acuerdo al nivel de riesgo del barrio, y la relación que existe entre el apoyo y control parental en los participantes que viven en zona de alto riesgo. En segundo lugar, se presenta el análisis de diferencias de las prácticas parentales respecto al sexo del participante que vive en una zona de alto riesgo. En tercer lugar, se detalla la relación que 
existe entre las prácticas parentales percibidas y el nivel de riesgo percibido por el adolescente que vive en zona de alto riesgo psicosocial.

En la tabla 1, respecto a la relación entre prácticas parentales y nivel de riesgo del entorno de residencia, se demuestra lo siguiente. En el contexto de alto riesgo, la figura materna muestra niveles significativamente más elevados que la figura paterna en lo que corresponde al factor afecto - comunicación $(Z=-2.701, p<.01)$, al factor crítica - rechazo $(Z=-4.557, p<.01)$ y al factor normas rígidas $(Z=-2.504, p<.02)$. En este caso, los adolescentes participantes perciben a la figura materna con una mayor presencia que el padre en lo que se refiere tanto a la comunicación afectuosa como a la comunicación crítica; y por consiguiente, en la manera rígida en que imponen las normas.

En lo que respecta al entorno de bajo riesgo, los resultados son los mismos, la figura materna muestra niveles significativamente más elevados que la figura paterna en los factores afecto - comunicación $(Z=-3.296, p<.01)$, crítica $-\operatorname{rechazo}(Z=-3.556, p<.01)$ y normas rígidas $(Z=-3.639, p<.01)$; sin embargo, la figura paterna muestra niveles significativamente más altos que la figura materna en lo que corresponde al factor de normas indulgentes $(Z=-3.798, p<.01)$, sobre el cual se entiende que la figura paterna es percibida, a diferencia de la figura materna, como una persona más permisiva en el momento de ejercer la imposición de las normas.

Tabla 1

Análisis de diferencias de las prácticas parentales de acuerdo a la figura parental

\begin{tabular}{ccccccccccc}
\hline \multicolumn{1}{c}{ Madre } & \multicolumn{9}{c}{ Padre } \\
Escalas & $M d n$ & $\begin{array}{c}M i \\
n\end{array}$ & Max & $M d n$ & $\begin{array}{c}M i \\
n\end{array}$ & Max & $Z$ & $p$ & $\begin{array}{c}d \text { de } \\
\text { Cohen }\end{array}$ & $1-\beta$ \\
\hline Alto R & & & & & & & & & & \\
FI & 39 & 13 & 50 & 34 & 10 & 50 & -2.701 & $<.01$ & .29 & .14 \\
FII & 16 & 11 & 43 & 15 & 10 & 44 & -4.557 & $<.01$ & .59 & .28 \\
F1 & 40 & 14 & 50 & 39 & 13 & 50 & -.841 & .40 & .03 & .02 \\
F2 & 32 & 14 & 45 & 31 & 12 & 50 & -2.504 & $<.02$ & .21 & .10 \\
F3 & 17 & 9 & 30 & 18 & 9 & 34 & -.989 & .32 & -.19 & -.10 \\
Bajo R & & & & & & & & & & \\
FI & 38 & 12 & 50 & 34 & 11 & 50 & -3.296 & $<.01$ & .33 & .16 \\
FII & 17.5 & 10 & 38 & 15.5 & 10 & 31 & -3.556 & $<.01$ & .56 & .27 \\
F1 & 39 & 18 & 49 & 37 & 12 & 50 & -.860 & .39 & .14 & .07 \\
F2 & 30 & 12 & 48 & 26 & 12 & 46 & -3.639 & $<.01$ & .41 & .20 \\
F3 & 16 & 9 & 28 & 18 & 9 & 34 & -3.798 & $<.01$ & -.48 & -.23 \\
\hline
\end{tabular}

Nota: FI=Factor afecto-comunicación, FII=Factor crítica-rechazo, F1=Factor normas inductivas, $\mathrm{F} 2=$ Factor normas rígidas, $\mathrm{F} 3=$ Factor normas indulgentes. $N=167$ 
En la tabla 2 se aprecia que la única diferencia significativa se encuentra en lo que corresponde a las normas rígidas percibidas de la figura paterna $(U=2493, p<.01)$. La figura paterna del entorno de alto riesgo muestra índices significativamente más elevados que la figura paterna de un entorno de bajo riesgo. En este sentido, los adolescentes que viven en una zona de alto riesgo psicosocial perciben a su figura paterna más predispuesta a imponer normas rígidas que sus pares que viven en un entorno de residencia de bajo riesgo.

Tabla 2

Análisis de diferencias de las prácticas parentales de acuerdo al nivel de riesgo del barrio

\begin{tabular}{ccccccccccc}
\hline \multicolumn{1}{c}{ Alto Riesgo } & \multicolumn{1}{c}{ Bajo Riesgo } \\
Escalas & Mdn & $\begin{array}{c}\text { Mi } \\
n\end{array}$ & Max & Mdn & $\begin{array}{c}\text { Mi } \\
n\end{array}$ & Max & $U$ & $p$ & $\begin{array}{c}d \text { de } \\
\text { Cohen }\end{array}$ & $1-\beta$ \\
\hline Madre & & & & & & & & & & \\
FI & 39 & 13 & 50 & 38 & 12 & 50 & 3281.5 & .51 & .10 & .10 \\
FII & 16 & 11 & 43 & 17.5 & 10 & 38 & 3139.5 & .28 & .18 & .20 \\
F1 & 40 & 14 & 50 & 39 & 18 & 49 & 3233.0 & .42 & .12 & .12 \\
F2 & 32 & 14 & 45 & 30 & 12 & 48 & 2990.5 & .11 & .24 & .33 \\
F3 & 17 & 9 & 30 & 16 & 9 & 28 & 3008.5 & .13 & .24 & .33 \\
Padre & & & & & & & & & & \\
FI & 34 & 10 & 50 & 34 & 11 & 50 & 3264.5 & .48 & .10 & .10 \\
FII & 15 & 10 & 44 & 15.5 & 10 & 31 & 3184.0 & .33 & .16 & .17 \\
F1 & 39 & 13 & 50 & 37 & 12 & 50 & 3162.0 & .30 & .16 & .17 \\
F2 & 31 & 12 & 50 & 26 & 12 & 46 & 2493.0 & $<.01$ & .52 & .90 \\
F3 & 18 & 9 & 34 & 18 & 9 & 34 & 3248.5 & .45 & .12 & .12 \\
\hline
\end{tabular}

Nota: FI=Factor afecto-comunicación, FII=Factor crítica-rechazo, F1=Factor normas inductivas, $\mathrm{F} 2=$ Factor normas rígidas, $\mathrm{F} 3=$ Factor normas indulgentes.

$N=167$

En esta misma línea de análisis y en relación entre el apoyo y el control parental en una zona de alto riesgo, la tabla 3 presenta la percepción de la figura materna, de la figura paterna y la relación entre ambas. En relación a la figura materna se observa que un aumento del nivel de afecto y comunicación se asocia negativamente con el nivel de crítica y rechazo percibido ( $r$ $=-.41, \mathrm{p}<.01)$ y que las normas inductivas y rígidas están directamente asociadas $(r=.30, \mathrm{p}<.01)$. Finalmente, se observa una asociación entre el nivel de afecto y comunicación con la percepción de vivenciar normas inductivas $(r=.73, \mathrm{p}<.01)$, y también con la percepción de vivenciar normas rígidas, aunque en menor medida $(r=.26, \mathrm{p}<.05)$. 
En relación a la figura paterna, se observa que un aumento del nivel de afecto y comunicación se asocia negativamente con los niveles de crítica y rechazo percibidos, de igual magnitud que en el caso de la figura materna $(r=-.40, \mathrm{p}<.01)$. Asimismo, las normas inductivas y rígidas percibidas también están directamente asociadas, aunque la asociación es más débil que en el caso de la figura materna $(r=.26, \mathrm{p}<.05)$. Finalmente, se aprecia una asociación significativa entre el nivel de afecto y comunicación con la percepción de vivenciar normas inductivas $(r=.66, \mathrm{p}<.01)$, indulgentes $(r=.34, \mathrm{p}<.01)$ y rígidas $(r=.29, \mathrm{p}<.01)$, de acuerdo a la intensidad de la relación.

En cuanto a la relación entre las dimensiones de ambas figuras, se puede apreciar que el nivel afecto y comunicación de la figura materna está relacionado con el mismo nivel de la figura paterna $(r=.32, \mathrm{p}<.01)$, de la misma manera que ocurre con el nivel de crítica y rechazo, aunque con mayor intensidad $(r=.49, \mathrm{p}<.01)$. Así como también se observa con alta intensidad que los tres modos de imponer normas de la figura materna se asocian con el respectivo modo de imponer normas de la figura paterna: normas inductivas $(r=.59, \mathrm{p}<.01)$, normas rígidas $(r$ $=.56, \mathrm{p}<.01)$ y normas indulgentes $(r=.53, \mathrm{p}<.01)$.

Tabla 3

Relación entre las figuras parentales en adolescentes de alto riesgo

\begin{tabular}{lcccccccccc}
\hline Escalas & 1 & 2 & 3 & 4 & 5 & 6 & 7 & 8 & 9 & 10 \\
\hline 1. FM-FI & ---- & & & & & & & & & \\
2. FM-FII &.$- .41^{* *}$ & ---- & & & & & & & & \\
3. FM-F1 & $.73^{* *}$ & $-.40^{* *}$ & ---- & & & & & & & \\
4. FM-F2 & $.26^{*}$ & .14 & $.30^{* *}$ & ---- & & & & & & \\
5. FM-F3 & .02 & .02 & .06 & .02 & ---- & & & & & \\
6. FP-FI & $.32^{* *}$ & .11 & $.31^{* *}$ & $.22^{*}$ & .13 & ---- & & & & \\
7. FP-FII & $-.24^{*}$ & $.49^{* *}$ & $-.26^{*}$ & .11 & .13 & $-.40^{* *}$ & ---- & & & \\
8. FP-F1 & $.44^{* *}$ & -.18 & $.59^{* *}$ & .20 & .09 & $.66^{* *}$ & $-.42^{* *}$ & ---- & & \\
9. FP-F2 & .13 & .15 & .16 & $.56^{* *}$ & .11 & $.29^{* *}$ & .16 & $.26^{*}$ & --- & \\
10. FP-F3 & -.10 & .16 & -.08 & .15 & $.53^{* *}$ & $.34^{* *}$ & .06 & .15 & .06 & --- \\
\hline
\end{tabular}

Nota: FM-FI=Figura materna - Factor afecto-comunicación, FM-FII=Figura materna - Factor críticarechazo, FM-F1=Figura materna - Factor normas inductivas, FM-F2=Figura materna - Factor normas rígidas, FM-F3=Figura materna - Factor normas indulgentes, FP-FI=Figura paterna - Factor afectocomunicación, FP-FII=Figura paterna - Factor crítica-rechazo, FP-F1=Figura paterna - Factor normas inductivas, FP-F2=Figura paterna - Factor normas rígidas, FP-F3=Figura paterna - Factor normas indulgentes. $N=85 ;{ }^{*} p<.05 ; * * p<.01$ 
En la tabla 4, sobre la relación entre prácticas parentales y género en un entorno de alto riesgo, se muestra que existen diferencias significativas en lo que corresponde al nivel de crítica y rechazo percibido de la figura materna $(U=575, p<.01)$ y de la figura paterna $(U=569, p<.01)$. En este caso, podemos inferir que los adolescentes varones que viven en una zona de alto riesgo perciben a ambas figuras parentales más críticas a comparación de las mujeres.

\section{Tabla 4}

Análisis de diferencias de las prácticas parentales de acuerdo al sexo del adolescente que vive en una zona de alto riesgo

\begin{tabular}{lcccccccccc}
\hline \multicolumn{1}{c}{ Mujeres } & \multicolumn{8}{c}{ Hombres } \\
Escalas & Mdn & $\begin{array}{c}\text { Mi } \\
n\end{array}$ & Max & Mdn & $\begin{array}{c}\text { Mi } \\
n\end{array}$ & Max & $U$ & $p$ & $d$ & $1-\beta$ \\
\hline Madre & & & & & & & & & & \\
FI & 39 & 13 & 50 & 38.5 & 19 & 49 & 813.5 & .63 & .10 & .07 \\
FII & 15 & 11 & 43 & 19 & 12 & 31 & 575.5 & .01 & .61 & .76 \\
F1 & 40 & 14 & 50 & 39 & 21 & 50 & 763.5 & .35 & .20 & .14 \\
F2 & 32 & 14 & 45 & 31 & 22 & 45 & 843.0 & .83 & .04 & .05 \\
F3 & 17 & 9 & 30 & 16 & 12 & 27 & 859.0 & .94 & .02 & .05 \\
Padre & & & & & & & & & & \\
FI & 33 & 10 & 50 & 34.5 & 14 & 50 & 798.0 & .54 & .14 & .09 \\
FII & 14 & 10 & 44 & 17 & 11 & 40 & 569.0 & .01 & .61 & .76 \\
F1 & 39 & 13 & 50 & 34.5 & 21 & 50 & 769.5 & .38 & .20 & .14 \\
F2 & 32 & 12 & 50 & 30.5 & 17 & 43 & 791.0 & .50 & .14 & .09 \\
F3 & 18 & 10 & 34 & 17 & 9 & 26 & 775.5 & .41 & .18 & .12 \\
\hline
\end{tabular}

Nota: FI=Factor afecto-comunicación, FII=Factor crítica-rechazo, F1=Factor normas inductivas, $\mathrm{F} 2=$ Factor normas rígidas, $\mathrm{F} 3=$ Factor normas indulgentes.

$N=85$

En la tabla 5 se puede apreciar que, en cuanto a la relación entre prácticas parentales y el nivel de riesgo percibido en los adolescentes que viven en una zona de alto riesgo, existe una tendencia que relaciona inversamente el nivel de riesgo percibido en el barrio con la predisposición de la figura materna a emplear normas rígidas $(r=-.23, \mathrm{p}<.05)$. 
Tabla 5

Relación entre prácticas parentales y el riesgo percibido en el barrio de alto riesgo

\begin{tabular}{cc}
\hline Escalas & Riesgo percibido \\
\hline Madre & -.09 \\
FI - Afecto-comunicación & .08 \\
FII - Crítica-rechazo & -.12 \\
F1 - Normas inductivas & $-.23^{*}$ \\
F2 - Normas rígidas & -.16 \\
F3 - Normas indulgentes & -.02 \\
Padre & -.01 \\
FI - Afecto-comunicación & -.15 \\
FII - Crítica-rechazo & -.08 \\
F1 - Normas inductivas & -.01 \\
F2 - Normas rígidas & \\
F3 - Normas indulgentes &
\end{tabular}

\section{Discusión}

En este capítulo se presenta el análisis e interpretación de los resultados obtenidos en la presente investigación. Para dicho fin, se describe y discute, en primer lugar, las prácticas parentales percibidas de los padres de un grupo de adolescentes que viven en una zona de alto riesgo psicosocial, para luego analizar el comparativo realizado con un grupo de adolescentes que viven en una zona no considerada de alto riesgo psicosocial. En segundo lugar, se discute las prácticas parentales percibidas de los padres de un grupo de adolescentes que viven en una zona de alto riesgo psicosocial de acuerdo al sexo del participante; para finalmente, analizar las mismas prácticas parentales bajo el nivel de riesgo percibido por los adolescentes.

Las prácticas parentales percibidas por adolescentes de su figura materna desde la perspectiva del apoyo parental están caracterizadas por una predominancia del afecto y la comunicación en desmedro de la crítica y el rechazo. Considerando ello, las muestras de afecto y comunicación en el ámbito intrafamiliar del Callao parecen resultar una tendencia que aún persiste debido a la evidencia de que se cuenta con familias principalmente nucleares (Plan Regional de Fortalecimiento de las Familias 2015-2021 de la Región Callao, 2015). Si bien se tiene en cuenta la alta tasa de madres que han vivenciado alguna experiencia de violencia y la alta tasa de madres con escolaridad básica inconclusa, entendemos que contamos con figuras maternas y/o paternas resilientes que en forma innata y/o aprendida logran expresarles a sus hijos el afecto y el interés de establecer una adecuada comunicación con ellos. Implícitamente 
les otorgan un valor significativo a las demostraciones de apoyo, a pesar de encontrarse en un entorno adverso cargado de violencia.

En lo que respecta a las normas y exigencias puestas en práctica por la figura materna en esta población, entendido como el control parental que ejerce la figura materna, se priorizan las formas inductivas. La percepción del predominio del afecto y la comunicación cimienta la percepción del adolescente de que es validada su opinión y reconocida sus necesidades. Sin embargo, que la percepción de normas rígidas demuestre por su parte una presencia significativa y que la forma indulgente que procede de la madre registre una puntuación baja, permite concluir que existiría una clara posición de parte de las figuras maternas a establecer normas, menguando la magnitud del autoritarismo gracias al vínculo establecido.

En este contexto, se observa que el nivel de afecto y comunicación percibido de la figura materna se asocia directa y fuertemente con la percepción de normas inductivas de su parte, y en menor medida, con la percepción de normas rígidas. Seguramente en un entorno de alto riesgo psicosocial, la percepción de apoyo gira en torno a una comunicación frecuente sobre la seguridad y el "deber ser" para evitar riesgos, estableciéndose lineamientos de conducta de parte de la madre permanentemente (Del Barrio et al., 2009; Cuellar, Jones \& Sterrett, 2015; Suen et al., 2019). He ahí que la asociación no se establezca con una posición indulgente de la madre. De la misma manera, se comprueba que el afecto y la comunicación percibida están inversamente asociados con la percepción de crítica y rechazo de la figura materna. Sirviendo como un catalizador de asertividad en la comunicación que fortalece el vínculo madre e hijo/a.

En lo que respecta a la figura paterna; la tendencia se mantiene. El nivel de afecto y comunicación percibido de la figura paterna se asocia directa y fuertemente con la percepción de normas inductivas de su parte. Los adolescentes que viven en una zona de alto riesgo psicosocial también perciben de su figura paterna principalmente afecto y comunicación. Su nivel de percepción de crítica y rechazo tampoco logra ser significativa e incluso por debajo de lo esperado; sin embargo, los resultados evidencian un nivel de afecto y comunicación menor al registrado por la figura materna. En este caso, la figura materna es la persona que está la mayor parte del tiempo con el hijo/a, incluso a solas con él/ella, a comparación del tiempo efectivo con el que cuenta la figura paterna. Compartir más tiempo implicaría mayores encuentros y desencuentros. Quizá sea esa la razón por la cual se le categoriza a la figura materna como el elemento de mayor afecto y al padre como el menos crítico.

La percepción que tiene el adolescente sobre la manera en que la figura paterna establece normas y exigencias es equivalente a lo que percibe de su figura materna. Principalmente un estilo inductivo de corrección que se muestra estricto al ejercer control parental. La menor 
proporción de tiempo que la figura paterna comparte con el hijo adolescente, a comparación del tiempo que comparte con la figura materna, parece ser más efectivo en cuanto a la fluidez de la comunicación. El espacio de encuentro con el padre parece resultar un momento de distención para el adulto y el hijo. Es así que, sin necesidad de mostrarse indulgente, la figura paterna consigue demostrar apoyo parental y asertivamente sustentar con ello el control que ejerce.

En el caso de la figura paterna, el nivel percibido de afecto y comunicación se asocia directamente con la percepción de las tres formas de establecimiento de normas y exigencias: inductiva, indulgente y rígida, respectivamente. El nivel de apoyo paterno tiene una relación directa con la forma inductiva en la medida en que le brinda al adolescente una presencia significativa en el momento de estructurar límites y establecer consecuencias ante su incumplimiento. Por su parte, el acuerdo es entendido por el adolescente como un espacio valioso de diálogo que lo hace sentir en una posición de mayor autonomía (Catemaxca, 2018; Cruz, Flores \& Jiménez, 2019; Espinosa, Mendoza \& Villalba, 2020). En el caso de la forma indulgente, se entiende que la figura paterna, a diferencia de la materna, se permite concesiones a un nivel de mostrarse permisivo ante ciertas transgresiones. Esta actitud es reconocida por el adolescente como una situación cotidiana que se da sólo con la figura paterna y que puede ser entendida como un acto comprensivo que aumente el nivel de confianza. Sobre la forma rígida, las normas impuestas drásticamente parece que el propio adolescente lo justifica basándose en la clara percepción de apoyo paterno que vivencia. Ante esto, asume cualquier actitud y conducta paterna correctiva como intervenciones que subjetivamente al adolescente le brinda seguridad (Del Barrio et al., 2009; Cuellar, Jones \& Sterrett, 2015; Suen et al., 2019).

Cabe precisar que, si bien ambas figuras cuentan con una percepción de apoyo parental cimentada en el afecto y la comunicación, la percepción de la figura materna establece una asociación directa con la percepción de normas inductivas paternas y en menor medida con la percepción de afecto y comunicación paterna. En tal sentido las expresiones de afecto y nivel de comunicación de la figura materna parecen generar un clima de armonía en la dinámica familiar que promueve la comunicación asertiva y con ello el entendimiento entre sus miembros. La participación conciliadora de la figura materna parece adquirir un alto nivel de influencia en la dinámica familiar. Por lo que se concluye que en el caso de los adolescentes que viven en una zona de alto riesgo psicosocial, la percepción del afecto y comunicación de la figura materna adquiere un valor predominante en el afán de fortalecer, no sólo el vínculo del adolescente con sus padres, sino el control efectivo que se ejerza sobre ellos.

Habiendo descrito y analizado las prácticas parentales desde el punto de vista de los hijos adolescentes que viven en una zona de alto riesgo psicosocial, se procederá a discutir el análisis 
comparativo con una muestra de adolescentes que viven una zona no considerada de alto riesgo psicosocial. El estudio nos indica que existe solo una diferencia significativa: la percepción de que la figura paterna de un entorno de alto riesgo emplea formas rígidas para establecer normas y exigencias es más frecuente, al percibido en una figura paterna de una zona no considerada de alto riesgo. Ahora bien, para lograr una interpretación acertada sobre la diferencia y entendiendo que las pruebas aplicadas no cuentan con índices que sugieran categorías, se observa que en Bersabé et al. (2001) las medianas obtenidas, en los modos rígidos de establecimientos de normas desde la perspectiva adolescente, se encuentran entre 26 y 29 puntos. Dicha observación nos sugiere que la figura paterna que vive en una zona de alto riesgo evidencia unas prácticas parentales dirigidas en mayor cuantía a la rigidez, en su modo de establecer normas y exigencias, a comparación de una figura paterna que vive en una zona no considerada de alto riesgo. La percepción de formas rígidas en la conducción de los límites es significativamente más frecuente en una zona de alto riesgo. Esta figura paterna intensifica su conducta hacia el autoritarismo como un recurso (in)consciente de adaptación al entorno de riesgo. La idea de inseguridad parece ser latente y con ello surge el temor inequívoco de estar sujetos a cierto nivel de riesgo que se compensa con restricciones.

En este sentido, cabe mencionar que tanto la figura materna que vive en una zona de alto riesgo como la que vive en una zona no considerada de riesgo, también demuestran esta predisposición en la misma cuantía. La sensación de inseguridad en ambas debe generar también una predisposición a manejar con mayor rigidez de la esperada las normas, por encima incluso de la figura paterna que vive en una zona de alto riesgo. Es así que la figura paterna que vive en una zona no considerada de alto riesgo es el único que demuestra en el estudio estar dentro del parámetro de rigidez comúnmente obtenido.

Finalmente, salvo la diferencia a nivel de formas rígidas que fue descrito en los párrafos anteriores, se destaca la similitud en las percepciones de los dos grupos adolescentes. Si bien la literatura científica al respecto no sugería una hipótesis de respuesta definida, se esperaba que la influyente variable zona de residencia establezca mayores diferencias. En efecto, intentando delimitar dos grupos que viva en zonas de residencia catalogadas como zona no considerada de alto riesgo y zona de alto riesgo, pero cuidando que compartan espacios, estímulos y aficiones similares; es que se previno cierta cercanía geográfica que probablemente no fue la suficiente para controlar la percepción paterna de inseguridad. En términos de normas y exigencias, la figura materna que vive en una zona no considerada de alto riesgo, sobre la cual los hijos adolescentes describieron sus prácticas parentales, ha demostrado ser una figura materna que se expresa como si viviera en una zona de alto riesgo. Mientras que, a nivel de afecto y 
comunicación, la similitud que se describe en ambos grupos sobre las dos figuras parentales nos hace concluir que en las familias del Callao existe una valiosa disposición al diálogo, que junto a la espontaneidad propia de la persona que vive en esa provincia se genera un clima armónico entre padres e hijos.

Como se indicó al iniciar el capítulo 4, corresponde analizar las diferencias de la percepción de prácticas parentales de acuerdo al sexo del adolescente que vive en una zona de alto riesgo. En tal sentido, se puede afirmar que los adolescentes varones significativamente perciben un mayor nivel de crítica y rechazo de parte de ambas figuras parentales.

Es necesario destacar que los resultados del presente estudio evidencian con mayor precisión que los adolescentes de ambos sexos perciben por igual la diferencia que existe de la figura materna respecto a la paterna en lo que se refiere a las demostraciones afectivas y grado de comunicación. Así, se reafirma que la figura paterna es la persona que menos apoyo parental transmite, de acuerdo a la percepción de adolescentes hombres y mujeres. Este hallazgo reafirma la propuesta teórica de esta investigación que, si bien existe una disposición en ambas figuras parentales de construir espacios de afecto y comunicación, se asume que la figura materna dispone de mayor tiempo efectivo para compartir con los hijos, y ello le brinda la oportunidad de conseguir un mejor posicionamiento frente a ellos y mayor relevancia (Del Barrio et al., 2009; Cuellar, Jones \& Sterrett, 2015; Suen et al., 2019).

En lo que respecta a los modos en que los adolescentes hombres y mujeres perciben que sus figuras parentales manejan las normas y exigencias, se encontró que no existe diferencia significativa. El sexo no es un condicionante para que alguna de las figuras parentales realice un trato diferenciado, a pesar que existe evidencia científica de que esperaría lo contrario. Esto probablemente se enmarca en el concepto internalizado de los padres de que el nivel de riesgo de la zona de residencia condiciona a ambos sexos a conducirse bajo las mismas normas. De esta manera, la presente investigación concibe que el principal determinante de la forma en que los padres imparten las normas sea el nivel de riesgo del entorno de residencia (Chetty, Hendren \& Katz, 2016; Madueño, Lévano \& Salazar, 2020; Newbury et al., 2016).

Por último, corresponde analizar la relación entre las prácticas parentales percibidas por adolescentes que viven en una zona de alto riesgo con respecto al nivel de riesgo que ellos mismos perciben. En este punto existe una tendencia que relaciona inversamente el nivel de riesgo percibido con un manejo rígido de las normas de parte de la figura materna. Cuan más rígida sea percibida la figura materna, se observa un menor riesgo percibido de su entorno, y viceversa. Considerando un marco intrafamiliar donde la madre representa el principal elemento de apoyo parental, la actitud impositiva y exigente materna instala en el adolescente 
una sensación inconsciente "de estar protegido", desestimando así los riesgos del entorno. Que la figura materna asuma un rol autoritario sería un modo de asumir y ejercer un liderazgo que establezca una ruta de acción siempre asumida como segura, al margen de no ser autónoma. Sería por ello que, cuando la percepción de normas rígidas disminuye, la percepción de riesgo aumenta en el adolescente, ya que en ese momento no percibe la directriz de la figura materna que lo conozca y pueda orientarlo.

Finalmente, en cuanto a las limitaciones del estudio podemos mencionar que convendría delimitar la muestra habiendo certificado el entorno de residencia del participante y no sólo considerar el centro de estudios al que asiste. Así mismo, hubiera resultado un aporte significativo el adaptar a nuestro contexto las escalas utilizadas. Y también, enriquecería el estudio lograr incorporar a los padres mediante la aplicación de la prueba en su versión para padres.

\section{Conclusiones}

En primer lugar, los adolescentes de una zona de alto riesgo psicosocial expresan, desde su percepción, que sus padres priorizan el afecto, el interés y la comunicación cuando ejercen ese rol, atribuyéndole especialmente al objeto materno dicha práctica. Asimismo, sus padres recurren al diálogo cuando deben de supervisar o exigir el cumplimiento de sus límites; sin embargo, en este punto cabe mencionar que a comparación del grupo control, evalúan a sus padres de manera más autoritaria, entendiéndolo como una medida compensatoria a la percepción de riesgo en el entorno de residencia, en tanto se evidencia que este matiz autoritario impacta positivamente en la sensación de seguridad que tiene el adolescente que se desarrolla en un entorno adverso. Finalmente, se demuestra que los varones, a comparación de las mujeres del estudio, son más susceptibles a las expresiones de los padres, en tanto perciben de ellos grados más altos de crítica y rechazo.

Ante lo expuesto, se sugiere que investigaciones posteriores puedan ahondar en la influencia que tiene el entorno de residencia en las prácticas parentales, desde la percepción de los propios padres, y cómo se relaciona ello con conductas de riesgo en adolescentes. Considerando, además, la opción de construir o adaptar un instrumento que permita explorar la percepción de niños sobre las prácticas parentales de sus padres, logrando obtener información que permita intervenir en la dinámica familiar de manera más temprana. 


\section{Referencias}

Álvarez-Malé, M., Bautista, I., \& Serra, L1. (2015). Prevalencia de los trastornos de la conducta alimentaria en adolescentes de Gran Canaria. Nutrición Hospitalaria, 31(5), 2283-2288. doi: $10.3305 /$ nh.2015.31.5.8583

Benarous, X., Guedj, M. J., de la Rivière, S. G., Guilé, J. M., \& Périsse, D. (2020). Conductas suicidas en la adolescencia. EMC-Tratado de Medicina, 24(3), 1-7. doi: 10.1016/S1636$\underline{5410(20) 44025-5}$

Benarous, X., \& Mazet, P. (2020). Trastornos psíquicos en la adolescencia. EMC-Tratado de Medicina, 24(1), 1-5. doi: 10.1016/S1636-5410(20)43347-1

Bernal, D. H., \& Bernal, L. P. (2020). Embarazo y adolescencia. Revista Cubana de Pediatría, 92(4). Recuperado de: http://www.revpediatria.sld.cu/index.php/ped/article/view/1290/636

Bersabé, R., Fuentes, M., \& Motrico, E. (2001). Análisis psicométrico de dos escalas para evaluar estilos educativos parentales. Psicothema, 13(4), 678-684. Recuperado de: http://www.psicothema.com/pdf/496.pdf

Bonilla, P. A. (2020). Impacto de la violencia infantil en los trastornos conductuales en la adolescencia. Ciencia Ecuador, 2(4), 8-13. Recuperado de: http://cienciaecuador.com.ec/index.php/ojs/article/view/20

Catemaxca, J. (2018). Prácticas parentales como predictoras de la conducta agresiva en adolescentes de una escuela secundaria en Montemorelos, NL (Tesis de licenciatura). Recuperado de: http://dspace.biblioteca.um.edu.mx/xmlui/handle/20.500.11972/402

Cruz, Y. C., Herrera, L. M. F., \& Flores, J. J. (2019). Prácticas parentales y ansiedad como predictoras del consumo de alcohol en adolescentes. Enseñanza e Investigación en Psicología, 55-64. Recuperado de: https://www.revistacneip.org/index.php/cneip/article/view/58/47

Cuellar, J., Jones, D.J. \& Sterrett, E. (2015) Examining Parenting in the Neighborhood Context: A Review. Journal of Child and Family Studies 24, 195-219. doi: 10.1007/s10826-0139826-y

Chetty, R., Hendren, N., \& Katz, L. (2016). The effects of exposure to better neighborhoods on children: New evidence from the moving to opportunity experiment. American Economic Review, 106(4), 855-902. doi: 10.1257/aer.20150572

Darling, N., \& Steinberg, L. (1993). Parenting style as context: An integrative model. Psychological Bulletin, 113(3), 487-496. doi: 10.1037/0033-2909.113.3.487

Del Barrio, V., Carrasco, M., Rodriguez, M., \& Gordillo, J. (2009). Prevención de la agresión en la infancia y la adolescencia. International Journal of Psychology and Psychological Therapy, 9(1), 101-107. Recuperado de: https://www.ijpsy.com/volumen9/num1/224/prevencin-de-la-agresin-en-la-infanciaES.pdf 
Díaz García, N. \& Moral Jiménez, M.V. (2018). Consumo de alcohol y conducta antiso-cial e impulsividad en adolescentes Españoles. Acta colombiana de Psicología, 21(2), 110 120. doi: $\underline{10.14718 / A C P .2018 .21 .2 .6}$

Duque, L., \& Klevens, J. (2000). La violencia en Itagüí, Antioquia: Prevalencia y distribución. Biomédica, 20, 151-168. Recuperado de: https://www.redalyc.org/articulo.oa?id=84320211

Espinosa, A., Mendoza, M., \& Villalba, V. (2020). Prácticas parentales en la regulación emocional de adolescentes. Revista Electrónica de Psicología de Iztacala. 23(1), 400417.

Recuperado

de: http://www.revistas.unam.mx/index.php/repi/article/view/75397/66767

Fresno, C. (2019). Metodología de la investigación. Córdoba, Argentina: El Cid Editor.

Fuentes, M. C., Alarcón, A., García, F., \& Gracia, E. (2015). Consumo de alcohol, tabaco, cannabis y otras drogas en la adolescencia: efectos de la familia y el barrio. Anales De Psicología, 31(3), 1000-1007. doi: 10.6018/analesps.31.3.183491

García Álvarez, D., Hernández-Lalinde, J., Espinosa-Castro, J. F., \& Soler, M. J. (2020). Salud mental en la adolescencia montevideana: una mirada desde el bienestar psicológico. Archivos Venezolanos de Farmacología y Terapéutica, 39(2), 182-190. Recuperado de: https://www.researchgate.net/publication/342453593_Salud_mental_en_la_adolescenci a_montevideana_una_mirada_desde_el_bienestar_psicologico

Gobierno Regional del Callao (2015). Plan Regional de Fortalecimiento de las Familias 20152021 de la Región Callao. Recuperado de http://observatoriodelasfamilias.mimp.gob.pe/normas/observatorionormatividadtodas.php

Gracia, E., Fuentes, M., \& García, F. (2010). Barrios de riesgo, estilos de socialización parental y problemas de conducta en adolescentes. Intervención Psicosocial, 19(3), 265-278. Recuperado de: $\quad$ http://scielo.isciii.es/scielo.php?script=sci $\operatorname{arttext\& pid=S1132-}$ $\underline{05592010000300007}$

Gracia, E., Herrero, J., Fuente, A., \& Lila, M. (2008). Neigborhood social disorder and attitudes toward partner violence against women: Evidence from Latin-american immigrants in Spain. The Open Sociology Journal, 1, 1-8. doi: 10.2174/1874946100801010001

Gracia, E., Herrero, J., Fuente, A., \& Lila, M. (2009). Perceived neighborhood social disorder and attitudes toward domestic violence against women among Latin-American inmigrants. The European Journal of Psychology Applied to Legal Context, 1(1), 25-43. Recuperado https://www.researchgate.net/publication/26644847_Perceived_neighborhood_social_di sorder and attitudes toward domestic violence against women among LatinAmerican immigrants

Ibarra, E. (2020). Tiempo con los amigos y la familia y el autoconcepto social y familiar durante la adolescencia. Revista de Psicología y Ciencias del Comportamiento de la Unidad 
Académica de Ciencias Jurídicas y Sociales, 11(1), 77-91. doi: 10.29059/rpcc.20200617$\underline{104}$

Martín-Fernández, M.; Matalí, J.; García-Sánchez, S.; Pardo, M.; Lleras, M.; CastellanoTejedor, C. (2017). Adolescentes con trastorno por juego en internet (IGD): perfiles y respuesta al tratamiento. Adicciones 29(2), 125-133. doi: 10.20882/adicciones.890

Mendoza, L., Claros, D. \& Peñaranda, C. (2016). Actividad sexual temprana y embarazo en la adolescencia: estado del arte. Revista chilena de obstetricia y ginecología, 81(3), 243253. doi: $10.4067 / \mathrm{S} 0717-75262016000300012$

Newbury, J., Arseneault, L., Caspi, A., Moffitt, T., Odgers, C., \& Fisher, H. (2016). Why are children in urban neighborhoods at increased risk for psychotic symptoms? Findings from a UK longitudinal cohort study. Recuperado de http://schizophreniabulletin.oxfordjournals.org/content/early/2016/05/05/schbul.sbw052 .full.pdf + html

Observatorio de la Criminalidad en el Callao (2015). El mapa del delito en el Callao. Recuperado de: http://larepublica.pe/723826-el-mapa-del-delito-en-el-callao

Sampson, R. J., \& Raudenbush, S. W. (1999). Assessing direct and indirect effects in multilevel designs with latent variables. Sociological Methods and Research, 28(2), 123-153. doi: $\underline{10.1177 / 0049124199028002001}$

Sampson, R. J., Raudenbush, S. W., \& Earls, F. (1997). Neighborhoods and violent crime: A multilevel study of collective efficacy. Science, 277, 918-924. doi: $\underline{10.1126 / \text { science. } 277.5328 .918}$

Suen, Y.; Cerin, E.; Barnett, A.; Huang, W. \& Mellecker, R. (2019) Associations of Sociodemographic, Family, and Neighborhood Factors with Physical Activity-Related Parenting Practices Among Hong Kong Preschoolers' Parents. Maternal and Child Health Journal, 23, 678-691. doi: 10.1007/s10995-018-2689-5

Wright, R., Mitchell, H., Visness, C., Cohen, S., Stout, J., Evans, R., \& Gold, D. (2004). Community violence and asthma morbidity: The inner-city asthma study. American Journal of Public Health, 94(4), 625-632. doi: 10.2105/AJPH.94.4.625 